\title{
Peer Reviewers for the Journal of the American Board of Family Medicine in 2017
}

\author{
Marjorie A. Bowman, MD, MPA, Anne Victoria Neale, PhD, MPH, \\ Dean Seehusen, MD, MPH, and Phil Lupo, MLIS
}

To our peer reviewers: We thank you for your time, expertise, and dedication to the fournal of the American Board of Family Medicine ( $7 A B F M)$.

$7 A B F M$ peer reviewers serve a vital role in contributing to the field of family medicine and to its scientific community. We take this opportunity to acknowledge and thank our peer reviewers for their support during the past year. In 2017, 409 individuals provided 528 peer reviews and advice regarding the suitability of articles for publication in the FABFM. Reviewers took an average of 19 days to submit a review.

\section{Top Reviewers}

Each of the manuscripts submitted to the $7 A B F M$ is first reviewed by the editors and, if deemed suitable

Conflict of interest: The authors are editors of the $7 A B F M$. for peer review, is evaluated and scored by volunteer reviewers. In the list below, peer reviewers with an asterisk next to their name are our top reviewers for 2017 based on evaluation scores and the quantity of critiques provided.

\section{Seeking New Reviewers}

We also enjoy welcoming new peer reviewers into the fold. Peer review is a vital service that helps authors and researchers improve their work. Reviewers are always free to decline a request or to indicate periods of unavailability. If you would like to volunteer to review for the FABFM, please download and complete the Peer Reviewer Form, which can be accessed online at http://jabfm.org/site/misc/PeerReviewForm. doc. Then, E-mail it, along with your curriculum vitae, to jabfm@med.wayne.edu, or fax it to 313577-9828. Thank you!

\begin{tabular}{lll}
\hline 2017 Peer Reviewers & & \\
Nahed Abdel-Haq & Matthew G Barnes & Alan Blum \\
Adrienne Z Ables & Donald Barr & Daniel S Blumenthal \\
Krishna A Agarwal & Kirsten Barrett & Debra Boardley \\
David C Agerter & Gillian Bartlett & George W Bock \\
Hector E Alcala & Laurence Bauer & Michael E Bodner \\
Sommer Aldulaimi & Dennis J Baumgardner & John M Boltri \\
Ayoob Ali & Elizabeth G Baxley & Curtis Bone \\
Thomas J Anan & Oralia V Bazaldua & Pranjal K Boruah \\
Kurt B Angstman & John W Beasley & Chantal Bouffard \\
Thomas A Arcury & Ian M Bennett & Rachel Bramson \\
Christine Arenson & Terrell Benold & Kent Bream \\
Terence J Aspray & George R Bergus & Fredrik F Broekhuizen \\
Russell T Attridge & Matthew Berman & Subbaraju Budharaju \\
Elisabeth L Backer & Ravi Bhargava & Christopher W Bunt \\
Steffani R Bailey & Juliann Binienda & Danielle Butler \\
Lodovico Balducci* & Rajshekhar Bipeta & Elena Byhoff \\
Laura-Mae Baldwin & Arthur Blain* & Linda M Cabral
\end{tabular}




\begin{tabular}{|c|c|c|}
\hline Doug Campos-Outcalt & Nancy C Elder* & Alexander B Guirguis \\
\hline Mollie Rose Canzona & Brian Elkins & Jacqueline R Halladay \\
\hline Roberto Cardarelli & John W Ely & Irene Hamrick \\
\hline Peter J Carek & John W Epling & E. Haneke \\
\hline Beth Careyva* & Ted Epperly & Ila M Harris \\
\hline Caroline S Carlin & James A Espinosa & James Harris \\
\hline A P Catinella & Rebecca S Etz & Robert L Hatch \\
\hline Andrew J Cave & Bernard G Ewigman & Clare Hawkins \\
\hline Joseph M Cerimele & Heather B Fagan & Jennifer Hefner \\
\hline William E Chavey & Krista Farey & Robin Helm \\
\hline Frederick M Chen & T M Farley & Margaret Helton \\
\hline Marcia J Chesebro & Timothy W Farrell & Sarah Hemeida \\
\hline Saleem Chowdhry & Steven R Feldman & David Henry \\
\hline Kaj S Christensen & Joshua J Fenton* & Paul J Hershberger \\
\hline Andreas Cohrssen & Jeanne $M$ Ferrante & Vincent W Hevern \\
\hline Allison M Cole & Robert L Ferrer & John M Hickner \\
\hline Cynthia Connelly & Scott A Fields & Vanessa Y Hiratsuka \\
\hline Colleen Conry & Edgar Figueroa* & Andrew Holdaway \\
\hline Steven D Crane & Alexander G Fiks & John Holmes \\
\hline Erica Crannage & Kenneth S Fink* & Christina T Holt \\
\hline Paul F Crawford & Kevin Fiscella & James D Holt \\
\hline Jesse C Crosson & Judith A Fisher & Eugene Hong \\
\hline Brian K Crownover & Kory Floyd & Steven Hooker \\
\hline Larry Culpepper* & Patricia Fontaine & Jennifer Horney \\
\hline Mike Cummings & Paul Fontelo & Rivka S Horowitz \\
\hline Amy Cunningham & Erika S Froelicher & Susan Hsieh \\
\hline Timothy P Daaleman & Kimberly G Fulda & Chun-Hsien Hsu \\
\hline Jessica Dalby & Heidi L Gaddey & Shawna V Hudson \\
\hline Jeanette M Daly & Anne H Gaglioti & William J Hueston \\
\hline Peter G Danis & Sim S Galazka* & Miranda M Huffman \\
\hline Robert J Darios & Charles A Gallia & Lauren S Hughes \\
\hline Jaividhya Dasarathy & Gregory M Garrison & Nathalie Huguet \\
\hline Alan K David & Sarah Gebauer & Sharon K Hull \\
\hline Philippa Davies & Dan Gelfman & Katherine Hurst \\
\hline Melinda M Davis & Christa George & Jamal Islam \\
\hline Dawn Davis* & Cynthia M Geppert & Henry R Ivey \\
\hline Matthew DeCamp & Jenenne Geske & Raja Jaber \\
\hline Frank deGruy & Abhijeet Ghatol & Muhammad A Javed \\
\hline Richard Derby & Valerie J Gilchrist & Anthony F Jerant \\
\hline Jennifer E DeVoe & Sarah L. Goff & Mark S Johnson \\
\hline Vanessa Diaz & Marji Gold & Monica Jolles \\
\hline L. Miriam Dickinson & Rachel Gold & Deborah Kahan \\
\hline Lorena Dini & Stephanie B Gold & Deepak Kamat \\
\hline Zora Djuric & Rise B Goldstein & Joy H Kang \\
\hline Mark P Doescher & Adam O Goldstein & Alan Katz \\
\hline Katrina E Donahue & Allen C Goodman & Deborah F Kehoe \\
\hline John N Dorsch & David T Grande & Drew M Keister \\
\hline Steven A Dosh & Bradley Gray & Rodger Kessler \\
\hline Chyke A Doubeni & Beverly Green & Vladimir Khanassov \\
\hline Tanya Dougherty & Lee A Green* & Niharika Khanna \\
\hline Dora M Dumont & Larry A Green* & Morteza Khodaee \\
\hline Milton Eder & Mark H Greenawald & Kim S Kimminau \\
\hline Jennifer Y Edgoose & Peter B Greenspan & Stella King* \\
\hline
\end{tabular}




\begin{tabular}{|c|c|c|}
\hline Dana E King & Raj Narayan & W. David Robinson \\
\hline Jeffrey T Kirchner & Susan Nash & Sean Robinson \\
\hline Christine Kistler & Donald E Nease & Adam J Rose \\
\hline Shae G Kosch & Lynne S Nemeth & Thomas C Rosenthal \\
\hline John Krueger & Dana Neutze & Steven E Roskos \\
\hline Robin L Kruse & Warren P Newton & Walter Rosser \\
\hline Irina Kudrina & Giang T Nguyen & Lisa Rubenstein \\
\hline Anton J Kuzel* & Bruce A Nitsche & Terry S Ruhl \\
\hline Maude Laberge & Thomas E Norris* & George Rust \\
\hline Bruce E Landon & Molly Nowels & Saria C Saccocio \\
\hline Sandi J Lane & Alane B O'Connor & Carolyn J Sachs \\
\hline Jason S Lanham & Thomas R O'Neill & Amanda H Salanitro \\
\hline Frank Lawler & LaDonna M Oelschlaeger & Kristian E Sanchack \\
\hline Ross A Lawrenson & Willie H Oglesby & Enrique Sanchez Valdivieso \\
\hline Aaron M Lear & Emily Onello & Paulo A Santos \\
\hline Lawrence M Leeman & Lynda J Owen & Ghanshyambhai T Savani \\
\hline Brian Lerner & Cristy Page & Barry G Saver \\
\hline Lawrence Leung & Scott A Paluska & Eric A Schaff \\
\hline Barcey T Levy & David Patchett & Carolina G Schlenker \\
\hline Winston Liaw & Himanshu Pathak & Miriam E Schwartz \\
\hline Cara Liday & Donald Pathman* & Thomas L Schwenk \\
\hline Andre F Lijoi & Dale A Patterson & Christopher N Sciamanna \\
\hline Sonja M Likumahuwa & Michael R Peabody & Kevin Selby \\
\hline Deerajnath Lingutla & Kevin A Pearce & Peter A Selwyn \\
\hline Mark Linzer & Randolph L Pearson & Patricia L Seymour \\
\hline Paula Darby Lipman & Deborah Peikes & Achal Sharma \\
\hline Everett E Logue & Rade N Pejic & Rajesh Sharma \\
\hline Margaret M Love & Allen Perkins & Eric K Shaw \\
\hline David A Loxterkamp* & Stephen Petterson & Michelle D Sherman \\
\hline Sean C Lucan & Lauren Peyton & Ranjit Singh \\
\hline Hillary D Lum & Lindsay Phillips & Jennifer N Smith \\
\hline Michael V Maciosek & William R Phillips & Robert Smith \\
\hline Michael K Magill & James D Plumb & Augustine J Sohn \\
\hline Rose C Maly & Lusine Poghosyan & Leif I Solberg \\
\hline Cara Marshall & Andrew Pomerantz & Samantha L Solimeo \\
\hline Eric Mast & Charles D Ponte & Jens Sondergaard \\
\hline Charles Maynard & Michael B Potter & Hassan Soubhi \\
\hline Ann S McAlearney & David Price & Linda M Spears \\
\hline Catherine McCarthy & James C Puffer & John B Standridge \\
\hline M. Diane McKee & Jon Puro & Nancy G Stevens \\
\hline Amy McQueen & Howard K Rabinowitz & Fiona Stevenson \\
\hline Ashley Meyer & Tiffany A Radcliff & Mark L Stovak \\
\hline Aaron Michelfelder & Melanie Raffoul & George D Strelioff \\
\hline Lloyd Michener & Francine Rainone & Larkin Strong \\
\hline Bryan Miller & Meghan F Raleigh & Denise K Sur \\
\hline William Miller & Ravishankar Ramaswamy & Andrew L Sussman \\
\hline Lynda Montgomery & Stephen Ratcliffe & John E Sutherland \\
\hline Victor M Montori & Brian V Reamy & Paul R Swank* \\
\hline Gerardo Moreno & Joanne L Reeve & Daniel J Tancredi \\
\hline Christopher P Morley* & Jo Marie Reilly & Hazel Tapp* \\
\hline Karoline Mortensen & Robert L Rhyne & Yhenneko J. Taylor \\
\hline Anne L Mounsey & Julie E Richards & Sharon Ternullo \\
\hline Karen Muchowski & Bradley A Richie & Chris M Terpening \\
\hline Zsolt J Nagykaldi & Lynne S Robins & Matthew Thompson \\
\hline
\end{tabular}




\begin{tabular}{lll}
\hline Barbara Tobias & Marlene M von Friederichs-Fitzwater & Calvin L Wilson \\
Sebastian T Tong & Gordon Walbroehl & Robin Winter \\
Stefan Topolski & Eric Wall* & Caroline M Wolff \\
Dennis Tsilimingras & John K Weiser & W Michael Woods \\
Carole C Upshur & Barry D Weiss & Imam M Xierali* \\
George Valko & Mary Jo Welker & Jinping Xu \\
Laura van Buul & Andrea Wendling & Mark J Yaffe \\
Constance van Eeghen & Randy K Wexler & Joseph R Yancey \\
Charles P Vega & John Sharkey White & Rodney B Young \\
Carla Veiga Rodrigues & Rachel Willard-Grace & Leanne Zakrzewski \\
William B Ventres & Summer Williams & Philip Zazove \\
Sarah Verbiest & Paul S Williamson & Emily B Zimmerman \\
Bonnie M Vest & &
\end{tabular}

*Top reviewers in 2017 (based on quantity and quality of peer reviews written).

To see this article online, please go to: http://jabfm.org/content/31/2/174.full. 